\title{
Energy-Efficient Subcarrier-and-Bit Allocation in Multi-User OFDMA Systems
}

\author{
Fourat Haider ${ }^{1}$, Cheng-Xiang Wang ${ }^{1}$, Harald Haas ${ }^{2}$, Erol Hepsaydir ${ }^{3}$, and Xiaohu Ge ${ }^{4}$ \\ ${ }^{1}$ Joint Research Institute for Signal and Image Processing, Heriot-Watt University, Edinburgh EH14 4AS, UK. \\ ${ }^{2}$ Joint Research Institute for Signal and Image Processing, University of Edinburgh, Edinburgh EH9 3JL, UK. \\ ${ }^{3}$ Hutchison 3G UK, 20 Grenfell Rd., Maidenhead, SL6 1EH, UK. \\ ${ }^{4}$ Department of Electronics and Information Engineering, Huazhong University of Science and Technology, Wuhan 430074, Hubei, China. \\ Email: \{fsh12, cheng-xiang.wang\}@hw.ac.uk, h.haas@ed.ac.uk, erol.hepsaydir@three.co.uk, xhge@mail.hust.edu.cn
}

\begin{abstract}
Energy efficiency is becoming increasingly important for wireless communication systems in order to minimize carbon footprint of wireless networks and to increase the battery life of mobile terminals. The spectral-energy efficiency trade-off is of primary significance to determine how much energy per bit is required in a wireless communication system to achieve a specific spectral efficiency. In this paper, we study energyefficient resource allocation scheme for Orthogonal FrequencyDivision Multiple Access (OFDMA) systems with multiple users. The trade-off between spectral and energy efficiencies is analyzed under the constraint of maintaining the fairness among users. We first formulate the energy-efficient optimization problem as an integer fractional programming. We then apply an iterative fractional method to simplify the problem to integer linear programming (ILP) problem. Simulation results demonstrate that the impact of user's quality of service $(\mathrm{QoS})$ is minor on the energy efficiency when a large spectral efficiency is required.
\end{abstract}

\section{INTRODUCTION}

The increasing number of new wireless devices and services leads to a significant increase in the demand for higher network capacity, higher user data rates, and new multimedia services in wireless networks. Although the convergence of mobile and internet puts more pressure on wireless service providers to offer faster and more efficient mobile internet access over limited resources, it comes at a high cost of higher energy consumption. Energy-efficient communication, also well-known as Green Radio [1], has attended a lot of research activities. The increase in energy consumption in wireless communication systems directly leads to the increase of $\mathrm{CO}_{2}$ emission, which is generally accepted as the major threat for the environment. Moreover, mobile operators have reported that more than $70 \%$ of their electricity power bills are mainly due to consumption by Radio Access Networks (RANs) [2]. Therefore, reducing energy consumption in RANs is important to minimize the carbon footprint of wireless networks. This leads to a significant reduction in the network operational cost and to the prolongation of battery life of mobile devices. Unfortunately, the joint optimization of energy efficiency and spectral efficiency is difficult to achieve as the improvement of one often is at the expense of the other. Therefore, it is important to investigate the different tradeoffs between the two performance indicators to decide upon the minimum energy consumption that is required to achieve the target spectral efficiency [3].

Radio resource allocation (RRA) in OFDMA systems has been extensively studied. Radio resources, i.e., orthogonal subcarriers and power, are allocated to different users to increase the performance of the system by use of different scheduling algorithms. It has been shown that the cell capacity improves as the number of simultaneous users increases in a cell due to multi-user diversity gain. Previous research on resource allocation in OFDMA-based systems mainly focused on either maximizing the number of bits per symbol subject to total transmit power, i.e., Rate Adaptive (RA) [4], or minimizing the total transmitted power subject to minimum data rate allocated to each user, i.e., Margin Adaptive (MA) [5]. However, neither RA or MA provides an energy-efficient solution. Recently, much efforts have been spent in developing energy-efficient resource allocation solutions for link-level Orthogonal Frequency Division Multiplexing (OFDM) systems [7]-[9]. Energy-efficient link level performance was studied for flat fading [6] and frequency-selective fading channels [7], [8]. The authors of [9] developed OFDM water-filling power allocation scheme that maximizes energy efficiency. Furthermore, some works have considered a muti-user scenario [10]-[12]. In [10], an energy-efficient power allocation scheme was proposed for interference-limited OFDMA systems. An algorithm was further developed in [11] that reduces the complexity with the iterative search technique developed in [10].

It is shown that the RRA problems for OFDMA systems are $(0,1)$ Linear programming (LP) in nature [14], [15]. None of the aforementioned works considered the use of ILP approach to solve the RRA problems with energy-efficient objectives. The ILP approach, however, can not be applied directly to energy-efficient optimization problems where it has to cope with fractional objective function. Therefore, in this paper, we first represent the objective function of the optimization problem by associated parametric single function in order to simplify the problem. Hereafter, we will adopt a similar approach to ILP as studied in [14], [15] and reformulate the non-linear energy-efficient optimization problem as ILP to find the optimum solution.

The rest of this paper is organized as follows. Section II 
gives a brief overview about energy efficiency metrics. Section III describes the system model adopted in this work. Section IV formulates the energy-efficient resource allocation problem. Section IV presents simulation results with detailed analysis. Finally, Section VI concludes the paper.

\section{Energy EFFiciency Metrics}

Energy efficiency (EE) has been defined in several ways [16]. One way is to take the ratio of the transmitted bit rate per unit time (or spectral efficiency) to the transmitted power (plus circuit power), measured in bits/Joule. The objective of this definition is to increase the number of transmitted information bits per unit energy which has been widely used in various publications [9]-[11]. However, as it was noticed recently in Energy Aware Radio and neTwork tecHnologies (EARTH) project [13], that using this definition for EE might lead to false conclusions especially at low power scenarios due to the lack of linearity between the consumed energy and transmitted power. A different way to define the EE is to use the energy consumption per bit, i.e., the ratio of consumed power per achievable rate (or spectral efficiency), as a metric measured in Joules/bit. The energy consumption increases linearly with transmitted power. The objective here is to minimize the total energy consumption for a given data rate. In this paper, we adopt the later definition.

\section{SySTEM MODEL}

In this paper a standard downlink OFDMA system is considered, as shown in Fig. 1, where a single Base Station (BS) is transmitting data towards a number of users utilizing a number of orthogonal sub-channels. Each sub-channel comprises of a group of subcarriers which are assumed to have correlated channel gains in frequency domain. The number of users and sub-channels are denoted by $n=1, \ldots, N$ and $k=1, \ldots, K$, respectively. The BS and users are all assumed to be equipped with a single antenna. It has been assumed that each subchannel which belongs to a particular user is under flat fading and corrupted by additive white Gaussian noise (AWGN). The channel state information (CSI) is sent to the BS over a feedback channel from each user. The CSI that represents a sub-channel is assumed to be equal to the linear average of all subcarriers' gain within that sub-channel. Furthermore, the BS has perfect CSI for each user instantly. Based on this information, the BS allocates a set of sub-channels to each user and decides on the number of bits in each sub-channel. It is assumed that each sub-channel is exclusively allocated to one user, i.e., sharing a sub-channel by two or more users is not allowed at any given time.

Adaptive modulation schemes with Binary Phase-Shift Keying (BPSK), Quadrature Phase-Shift Keying (QPSK), 16 Quadrature Amplitude Modulation (16-QAM), and 64-QAM are adopted to achieve a target Bit-Error Rate (BER). The information regarding subcarrier assignment and bit loading is sent to users over a control channel. The transmitted power for user $n$ on sub-channel $k$ is decided according to the target BER and the received power level. In other words, the transmitted power, normalized to background noise, can be expressed as

$$
P_{n, k}(\mathrm{BER}, c)=\frac{f\left(\mathrm{BER}, c_{n, k}\right)}{\left|h_{n, k}\right|^{2}}
$$

where $h_{n, k}$ is the complex-valued channel gain that the $n$th user experiences on the sub-channel $k . f\left(c_{n, k}\right)^{1}$ is the required received power level that depends on the modulation scheme, and $c_{n, k}$ is defined as the number of loaded bits on the $k$ th sub-channel sent to user $n$ over one OFDM symbol. It takes a value from the an integer nonnegative vector $\mathbf{D}=S \times\left\{0, b_{1} \ldots, b_{M}\right\} \in \mathbb{Z}^{M \times 1}$. Here, $S$ is the number of bits in each sub-channel and $M$ is the number of possible modulation schemes. Indeed, $M$ depends on the maximum supported modulation scheme that the BS can adapt. For BPSK and QPSK, $f\left(c_{n, k}\right)$ equals to $\frac{S}{2}\left(Q^{-1}(\mathrm{BER})\right)^{2}$ and $S\left(Q^{-1}(\mathrm{BER})\right)^{2}$, respectively [17], where $Q^{-1}$ is the inverse $\mathrm{Q}$ function. For the higher modulation schemes, i.e., 16-QAM and 64-QAM, the required received power can be calculated by [17]

$$
f\left(c_{n, k}\right)=\frac{S \times 2^{b}}{3}\left(Q^{-1}\left(\frac{b \times \mathrm{BER}}{4}\right)\right)^{2} .
$$

\section{ENERGY-EFFICIENT RESOURCE ALLOCATION}

In this section, the problem of energy-efficient subcarrier and bit allocation subject to some constraints will be formulated. Hereafter, an iterative approach will be adopted to achieve the optimum solution.

\section{A. Problem Formulation}

We define the EE as the energy required to transmit information data reliably within one OFDM symbol, measured in Joules/bit and the spectral efficiency (SE) as the total transmitted bits in one OFDM symbol measured in bits/OFDM symbol (or equivalently bps/Hz). Let's define $d_{n, k}$ as a binary indicator variable which is equal to one if the subcarrier $k$ is allocated to user $n$ and zero otherwise. The energy-efficient resource allocation problem can be expressed as

$$
\begin{array}{ll} 
& \min _{c_{n, k}, d_{n, k}} \mathrm{EE}=\frac{P_{\mathrm{C}}+\sum_{n=1}^{N} \sum_{k=1}^{K} P_{n, k} d_{n, k}}{\sum_{n=1}^{N} \sum_{k=1}^{K} c_{n, k} d_{n, k}} \\
\text { s.t. } \quad & \sum_{n=1}^{N} \sum_{k=1}^{K} c_{n, k} d_{n, k} \geq \mathrm{SE} \\
& \sum_{k=1}^{K} c_{n, k} d_{n, k} \geq \mathrm{R}_{n}^{\min } \\
& \sum_{n=1}^{N} \sum_{k=1}^{K} P_{n, k} d_{n, k} \leq P_{\mathrm{T}} \\
& \sum_{n=1}^{N} d_{n, k}=1 \\
& d_{n, k} \in\{0,1\}
\end{array}
$$

\footnotetext{
${ }^{1}$ For notational convenience, the BER index will be omitted in $f($.$) and$ $P_{n, k}($.$) through the rest of the paper since the value of BER is fixed in this$ work.
} 
where $P_{\mathrm{C}}$ is a circuit power dissipation that is assumed to be constant in this work. Constraint (4) ensures a minimum required SE. Constraint (5) ensures to obtain each user's QoS. $P_{\mathrm{T}}$ is the total power constraint. Constraint (7) means that each sub-channel is allocated to one user only. The aim in the above problem is to minimize the transmitted energy per bit for a given SE and to satisfy each user's QoS requirement as well.

Let's define a variable binary vector $\mathbf{X}$ as $\left[\begin{array}{llll}\mathbf{x}_{1} & \mathbf{x}_{2} & \ldots\end{array}\right.$ $\left.\mathbf{x}_{K}\right]^{T} \in\{1,0\}^{K N M \times 1}$, where $\mathbf{x}_{k}=\left[\begin{array}{llll}\mathbf{x}_{1}^{k} & \mathbf{x}_{2}^{k} & \ldots & \mathbf{x}_{N}^{k}\end{array}\right]^{T} \in$ $\{1,0\}^{N M \times 1}$ is the allocation vector that allocates a subchannel $k$ to a user $n$. Now, $\mathbf{x}_{n}^{k}=\left[\begin{array}{llll}x_{1}^{k, n} & x_{2}^{k, n} \ldots & x_{M}^{k, n}\end{array}\right]^{T} \in$ $\{1,0\}^{M \times 1}$ is the modulation vector that decides which modulation index that user $n$ is going to use if he assigned to sub-channel $k$. Also, let's define a power vector $\mathbf{P}$ as $\left[\begin{array}{lll}\mathbf{p}_{1} & \mathbf{p}_{2} \ldots & \mathbf{p}_{K}\end{array}\right] \in \mathbb{R}^{1 \times K N M}$, where $\mathbf{p}_{k}=\left[\begin{array}{llll}\mathbf{p}_{1}^{k} & \mathbf{p}_{2}^{k} & \ldots & \mathbf{p}_{N}^{k}\end{array}\right]$ $\in \mathbb{R}^{1 \times N M} \cdot \mathbf{p}_{n}^{k}=\left[\frac{f(1)}{h_{n, k}^{2}} \frac{f(2)}{h_{n, k}^{2}} \ldots \quad \frac{f(M)}{h_{n, k}^{2}}\right] \in \mathbb{R}^{1 \times M}$ represents the possible transmitted power. Likewise, a vector $\mathbf{C}$ which denotes the transmit bits in each sub-channel is defined as $\mathbf{C}=$ $\left[\begin{array}{llll}\mathbf{c}_{1} & \mathbf{c}_{2} & \ldots & \mathbf{c}_{K}\end{array}\right] \in \mathbb{Z}^{1 \times K N M}$, where, $\mathbf{c}_{k}=\left[\begin{array}{llll}\mathbf{D}_{1} & \mathbf{D}_{2} & \ldots & \mathbf{D}_{N}\end{array}\right]$ $\in \mathbb{Z}^{1 \times N M}$. Furthermore, we relax the constraint (7) to make the summation equal to either 0 or 1 because, for some channel realizations, it is more energy-efficient to not assign the subchannel $k$ to any user. By using these vectors definition, the optimization problem (3)-(8) can be reformulated to

$$
\begin{array}{ll} 
& \min _{x} \mathrm{EE}=\frac{\left(P_{\mathrm{C}}+\mathbf{P X}\right)}{\mathbf{C X}} \\
\text { s.t. } & -\mathbf{C X} \leq-\mathrm{SE} \\
& -\mathbf{A}_{u} \cdot \mathbf{X} \leq-\mathbf{R}^{\min } \\
& \mathbf{P X} \leq P_{\mathrm{T}} \\
& \mathbf{A}_{e} \cdot \mathbf{X} \leq \mathbf{1}^{T} \\
& \mathbf{X} \in \Omega \subseteq\{0,1\}
\end{array}
$$

where $\mathbf{A}_{u}=\left[\begin{array}{lll}\mathbf{a}^{1} \mathbf{a}^{2} \ldots & \mathbf{a}^{N}\end{array}\right]^{T} \in \mathbb{Z}^{N \times K N M}, \mathbf{a}^{n}=\left[\begin{array}{ll}\mathbf{a}_{1}^{n} & \mathbf{a}_{2}^{n} \ldots\end{array}\right.$

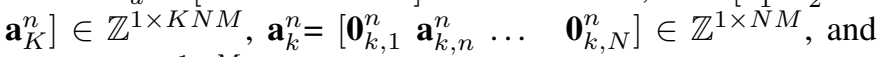
$\mathbf{a}_{k, n}^{n}=\mathbf{D} \in \mathbb{Z}^{1 \times M}$. Note that

$$
\mathbf{A}_{e}=\left[\begin{array}{lllc}
\mathbf{1}_{1,1} & \mathbf{0}_{1,2} & \ldots & \mathbf{0}_{1, K} \\
\mathbf{0}_{2,1} & \mathbf{1}_{2,2} & \ldots & \mathbf{0}_{2, K} \\
\vdots & \vdots & \ddots & \vdots \\
\mathbf{0}_{K, 1} & \mathbf{0}_{K, 2} & \cdots & \mathbf{1}_{K, K}
\end{array}\right]
$$

$\in\{1,0\}^{K \times K N M}$ is to ensure that sub-channel $k$ is either allocated exclusively to one user or excluded from the allocation. Here $\mathbf{1}_{k, k} \in\{1\}^{1 \times N M}$ and $\mathbf{0} \in\{0\}^{1 \times N M} . \mathbf{R}^{\min }=\left[\mathrm{R}_{1}^{\min } \mathbf{R}_{2}^{\min }\right.$ $\left.\ldots \mathrm{R}_{N}^{\min }\right]^{T}$ is the minimum rate vector to ensure each user's QoS and $\Omega$ is the feasible region for the described problem (9)-(14).

The objective function (9) is the ratio of two functions to be minimized. This type of optimization problem is commonly known as fractional programming [18]. This optimization problem can be more tractable by adopting Dinkledbach's parametric approach [19] as we will see in the following subsection.

\section{B. Problem Transformation and Parametric Algorithm}

Consider the following general fractional programming problem

$$
\begin{array}{ll}
\text { (P) } & \min _{x} Q(x)=\frac{f(x)}{g(x)} \\
\text { s.t. } & h(x) \geq 0, \quad x \in X
\end{array}
$$

where $X$ is a nonempty feasible set. Using a parametric approach in [19], the above fractional optimization problem $(P)$ can be solved indirectly by finding the solution to the following associated non-fractional optimization problem. i.e.,

$$
\begin{array}{ll}
(\bar{P}) \quad \min _{x} \bar{Q}(x)=f(x)-q g(x) \\
\text { s.t. } & h(x) \geq 0, \quad x \in X
\end{array}
$$

where $q \in R^{+}$is a constant. The problem $(\bar{P})$ is sometimes easier to solve than solving the problem $(P)$.

Theorem 1. Solving problem $(P)$ is equivalent to obtaining $q^{*}$ that makes $\bar{Q}(x)=0$, and thus $q^{*}=\frac{f\left(x^{*}\right)}{g\left(x^{*}\right)}$ is the optimum solution.

Proof: See Appendix A

Dinkelbach also proposed an iterative algorithm that solves the parametric problem, i.e, $(\bar{P})$. The algorithm generates a sequences of $q$ converging to $q^{*}$, as it is described in [19]. The algorithm terminates once the objective value of the problem $\bar{P}$ becomes zero. It is worth mentioning that Dinkelbach's method is still valid for fractional problems with objectives being maximized.

\section{Energy-Efficient Algorithm}

Applying a similar approach to the energy-efficient optimization problem, one can see that $f(x) \equiv\left(P_{\mathrm{C}}+\mathbf{P X}\right)$ and $g(x) \equiv \mathbf{C X}$. The EE in this case will be equivalent to $q^{*}$. This inspires us to propose an energy-efficient allocation algorithm which is summarized in Algorithm 1. The complexity of the

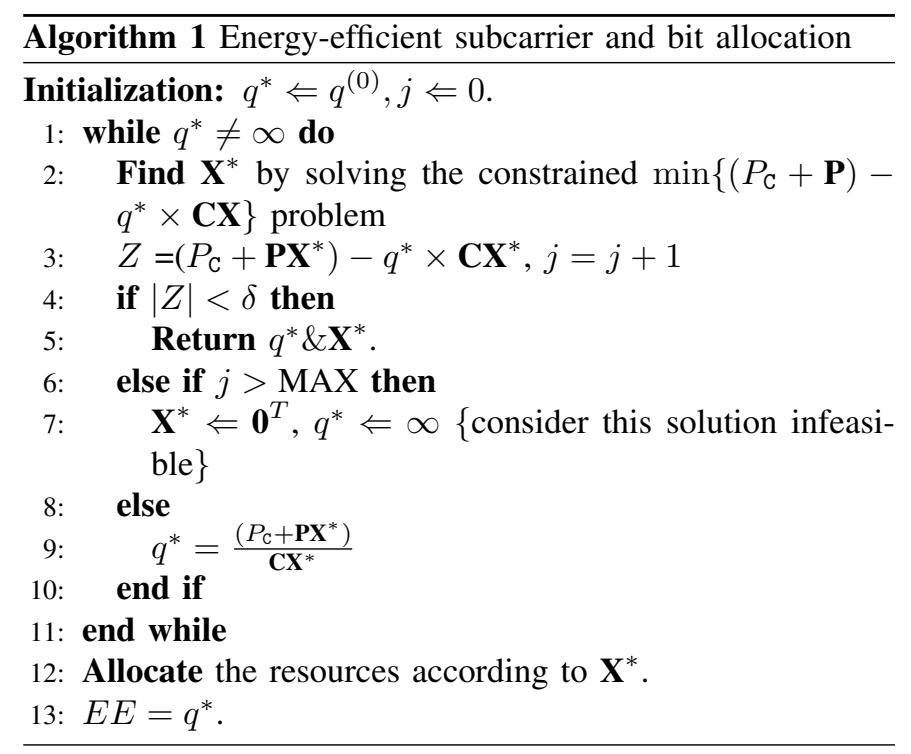

algorithm depends completely on the optimization problem 
solver in Step 2 and how it takes to converge to $q^{*}$. Hence, any binary LP-based solver, e.g., Branch-and-Bound $(\mathrm{BaB})$, can be used in Step 2 to solve the optimization problem $(\bar{P})$ for a given $q$.

\section{Simulation Results and Discussions}

This section evaluates the proposed energy-efficient algorithm and presents the simulation results. The simulator has a single cell with a radius of $1 \mathrm{~km}$. Long Term Evolution (LTE) frame structure, as standardized by 3 GPP Release 8 , is adopted with blocks of twelve contiguous subcarriers in frequency domain and fourteen OFDM symbols in the time domain [21]. Hence, the data transmissions are scheduled every $1 \mathrm{~ms}$. The simulation results presented in this paper are averaged over 1000 different channel conditions. The bandwidth of each sub-channel is equal to $180 \mathrm{kHz}$. The channels are subject to path-loss, log-normal shadowing with standard deviation of 8 $\mathrm{dB}$, and a frequency-selective fading with 6 taps. The path loss model is calculated by [20],

$$
L[\mathrm{~dB}]=136+37.6 \times \log _{10}(d)
$$

where $d$ is the distance between a user and the BS, in $\mathrm{km}$. The BER is fixed to $10^{-4}$ in all examples. The circuit power $P_{\mathrm{C}}$ is $100 \mathrm{Joules} / \mathrm{symbol}$.

Fig. 2 presents the spectral-energy trade-off under different user's QoS requirements. The number of sub-channels is 15 and shared by 4 users. All users are located at the cell edge. The total transmitted power is equal to 30 Joules/symbol. As it can be shown more energy is required as the minimum rate for each user increases especially at low SE. However, the gap tends to be small at high SE because the constraint on the SE is dominant and can satisfy the user's QoS requirement.

Fig. 3 depicts the EE as a function of the number of active users in the cell. The number of sub-channels is assumed to be 32 and $R^{\min }$ is equal $1 \mathrm{Mbps}$. Here, the users are being randomly dropped in each snapshot. As it is clearly shown, increasing the number of users results in decreasing the consumed energy per bit due to users diversity gain. It can also be noticed that with a larger number of users, a smaller amount of energy is required to double the SE.

Fig. 4 compares the EE as a function of the distance between the BS and users for two SEs and different user's QoS requirements. The number of users and the number of sub-channels are assumed to be 5 and 20 respectively. The EEs are approximately the same for the two SEs when the users are near the BS. The difference between EEs for the two SEs increases when the users is far from the BS since a full power is transmitted in the case of the highest SE.

\section{CONCLUSIONS}

In this paper, we have studied sub-channel and bit allocation scheme to minimize the energy per transmitted bit for multiusers in an OFDMA system. Simulation results has shown that the user's QoS requirement has a minor impact on the $\mathrm{EE}$ if large SE is needed. An optimization problem with EE objective function can be reformulated to a non-fractional function and solved using an iterative algorithm. This reduces the complexity of the original problem. The algorithm can also be applied to the energy-efficient optimization problem with an objective of maximizing the number of bits per joule.

\section{APPENDIX A \\ PRoOF OF THEOREM 1}

Let the vector $\mathbf{X}^{*}$ is the the soultion for problem $P$, then we have

$$
q^{*}=\frac{f\left(x^{*}\right)}{g\left(x^{*}\right)} \leq \frac{f(x)}{g(x)}, \quad \forall x \in \mathbf{X}
$$

this leads to

$$
f(x)-q^{*} g(x) \geq 0
$$

and concludes a fact that

$$
\min _{x}\left\{f(x)-q^{*} g(x)\right\}=0 .
$$

Now if the $x^{*}$ is the optimum solution, then the following is right:

$$
f(x)-q^{*} g(x) \geq f\left(x^{*}\right)-q^{*} g\left(x^{*}\right)=0
$$

which proves Theorem 1.

\section{ACKNOWLEDGMENTS}

This work is partially supported by Hutchison $3 \mathrm{G}$ in the UK. The authors would like to acknowledge the support from the RCUK for the UK-China Science Bridges Project: R\&D on (B)4G Wireless Mobile Communications. F. Haider, C.-X. Wang, and H. Haas would also like to acknowledge the support from the Scottish Funding Council for the Joint Research Institute in Signal and Image Processing between the University of Edinburgh and Heriot-Watt University, as part of the Edinburgh Research Partnership in Engineering and Mathematics (ERPem). The work of C.-X. Wang was also supported by the Opening Project of the Key Laboratory of Cognitive Radio and Information Processing (Guilin University of Electronic Technology), Ministry of Education (Grant No. 2011KF01). X. Ge acknowledges the support from the National Natural Science Foundation of China (NSFC) (Grant No.: 60872007), National 863 High Technology Program of China (Grant No.: 2009AA01Z239), and the Ministry of Science and Technology (MOST), China, International Science and Technology Collaboration Program (Grant No.: 0903) and Hubei Provincial Science and Technology Department (Grant No.: 2011BFA004).

\section{REFERENCES}

[1] C. Han, T. Harrold, S. Armour, I. Krikidis, S. Videv, P. M. Grant, H. Haas, J. S. Thompson, I. Ku, C.-X. Wang, T. A. Le, M. R. Nakhai, J. Zhang, and L. Hanzo, "Green radio: radio techniques to enable energy efficient wireless networks," IEEE Commun. Magazine, vol. 49, no. 6, pp. 46-54, June 2011.

[2] T. Edler and S. Lundberg, "Energy efficiency enhancements in radio access networks," in Erricsson Review, 2004.

[3] F. Haider, C.-X. Wang, X. Hong, H. Haas, D. Yuan, and E. Hepsaydir, "Spectral-energy efficiency tradeoff in cognitive radio networks with peak interference power constraints," invited paper, Proc. IEEE ICCT'11, Jinan, China, Sept. 2011.

[4] J. Jang and K. B. Lee, "Transmit power adaptation for multiuser OFDM systems," IEEE Trans. Select. Areas Commun., vol. 21, no. 2, pp. 171178, Feb. 2003 


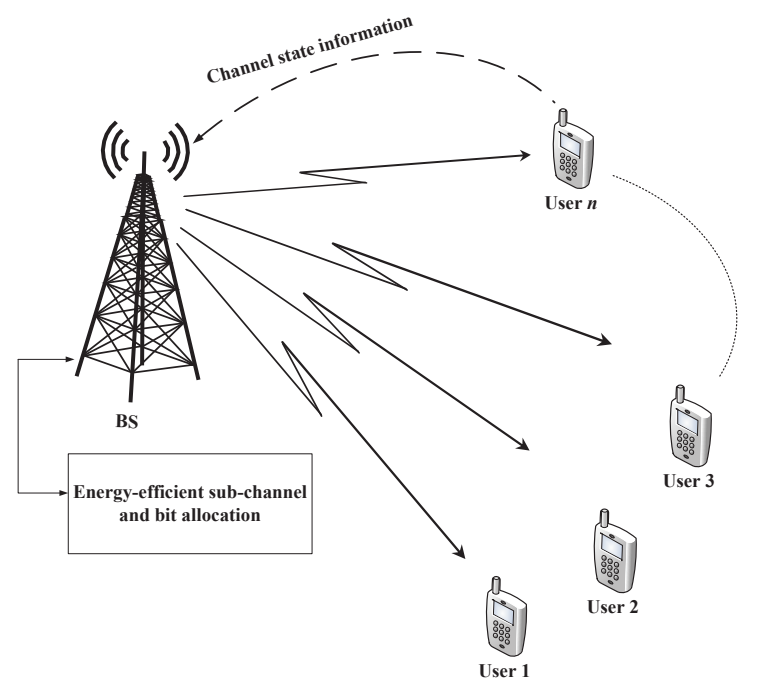

Fig. 1. System model.

[5] C. Y. Wong, R. S. Cheng, K. B. Letaief, and R. D. Murch, "Multiuser OFDM with adaptive subcarrier, bit and power allocation," IEEE Trans. Select. Areas Commun., vol. 17, pp. 1747-1758, Oct. 1999.

[6] G. W. Miao, N. Himayat, G. Y. Li, and D. Bormann, "Energy-efficient design in wireless OFDMA," in Proc. IEEE ICC'08, May 2008, pp. 33073312.

[7] G. W. Miao, N. Himayat, and G. Y. Li, "Energy-efficient transmission in frequency-selective channels," in Proc. IEEE GLOBECOM'08, Nov. 2008.

[8] C. Isheden and G. P. Fettweis, "Energy-efficient multi-carrier link adaptation with sum rate-dependent circuit power", in Proc. IEEE GLOBECOM'10, Dec. 2010.

[9] R. S. Prabhu and B. Daneshrad, "An energy-eficient water-filling algorithm for OFDM systems," in Proc. IEEE ICC'10, May 2010.

[10] G. Miao, N. Himayat, Y. Li, A. T. Koc, and S. Talwar, "Interferenceaware energy-efficient power optimization," in Proc. IEEE ICC'09, Dresden, Germany, June 2009, pp. 14-18.

[11] G. W. Miao, N. Himayat, and Y. Li, "Low-complexity energy-efficient OFDMA," in Proc. IEEE ICC '09, Dresden, Germany, June 2009.

[12] C. Xiong, G. Y. Li, S. Zhang, Y. Chen, and S. Xu, "Energy-and spectral- efficiency tradeoff in downlink OFDMA networks", in Proc. IEEE ICC'11, Atlanta, USA June 2011.

[13] G. Auer, V. Giannini, I. Godor, P. Skillermark, M. Olsson, M.A. Imran, D. Sabella, M.J. Gonzalez, C. Desset, and O. Blume, "Cellular energy efficiency evaluation framework," in Proc. IEEE VTC'11-Spring, Budapest, Hungary, May 2011.

[14] I. Kim, I. Park, and Y. Lee, "Use of linear programming for dynamic subcarrier and bit allocation in multiuser OFDM," IEEE Trans. Veh. Techn., vol. 55, no. 4, pp. 1195-1207, July 2006.

[15] Z. Mao and X. Wang, "Efficient optimal and suboptimal radio resource allocation in OFDMA system," IEEE Trans. Wireless Commun., vol. 7, no. 2, Feb. 2008.

[16] T. Chen, H. Kim, Y. Yang, "Energy efficiency metrics for green wireless communications," in Proc IEEE WCSP'10, Oct. 2010.

[17] A. Goldsmith,“ Wireless communications", Cambridge University Press, 2005.

[18] E. Bajalinov, " Linear-fractional programming: theory, methods, applications and software," Springer, 2003.

[19] W. Dinkelbach, "On nonlinear fractional programming", Management Science, vol. 13, pp. 492-498, 1967.

[20] J. Laiho, A. Wacker, T. Novosad,"Radio network planning and optimisation for UMTS", John Wiley \& Sons, Chichester, 2002.

[21] 3GPP, TR 25.814 V7.1.0, "Physical layer aspects for evolved UTRA" Rel. 7, Sep. 2006.

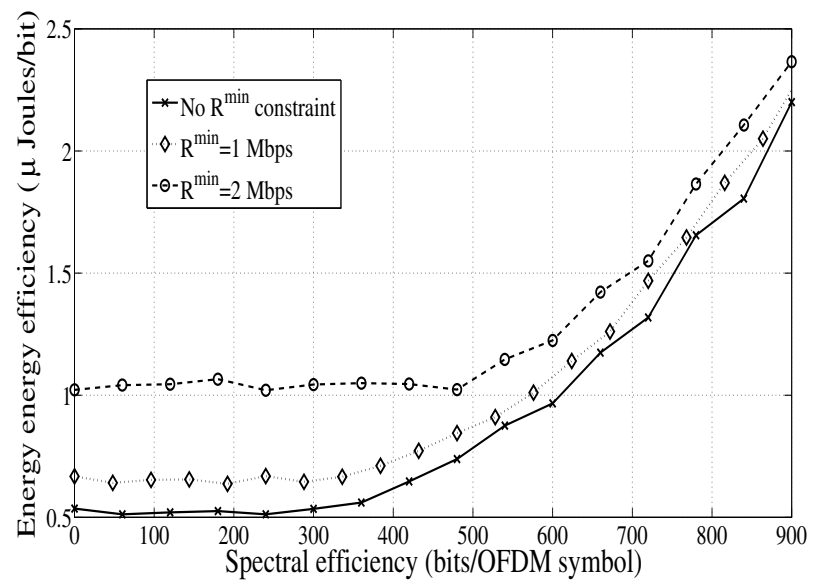

Fig. 2. System spectral-energy efficiency trade-off.

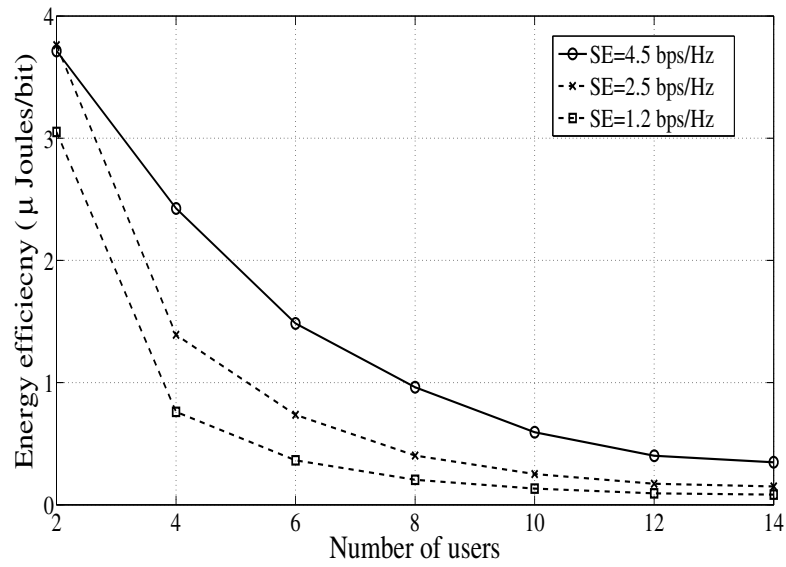

Fig. 3. Energy efficiency vs. the number of users.

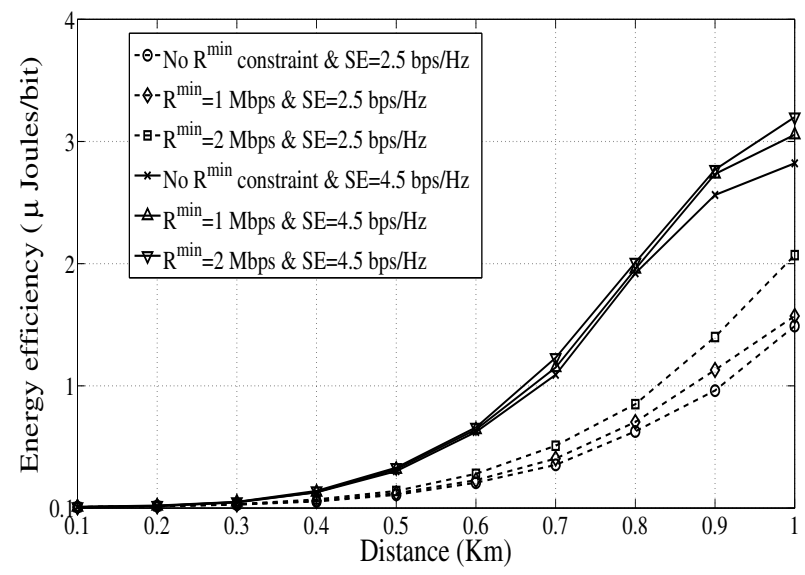

Fig. 4. Energy efficiency vs. the distance between the BS and users. 